\title{
Olympiads in Informatics in Republic of Moldova
}

\author{
Anatol GREMALSCHI ${ }^{1}$, Angela PRISACARU ${ }^{2}$, Sergiu CORLAT ${ }^{3}$ \\ ${ }^{1}$ Institute for Public Policy, Chisinau, Republic of Moldova \\ ${ }^{2}$ Department of Pre-University Education, Ministry of Education, Republic of Moldova \\ ${ }^{3}$ Department of Informatics and Information Technologies, Tiraspol State University, \\ Chisinau, Republic of Moldova \\ e-mail:anatol_gremalschi@ipp.md,aprisacaru@gmail.com,sergiu.corlat@math.md
}

\begin{abstract}
This article presents the retrospective of organizing the Republican Olympiad in Informatics from the first editions. It describes the current model of organizing the competition: steps, target groups, methodologies of development, evaluation, and the preparation of participants for the different stages of the competition.
\end{abstract}

Keywords: informatics curricula, IOI, olympiad in informatics, teaching programming.

\section{The History}

Informatics was introduced into the undergraduate studies in 1985. From the beginning, the curriculum was oriented to systematic studying the basic computing concepts: the structure of information and the basic principles of programming. This allowed the placement of informatics in the science field. Consequently, the discipline has benefited from lots of opportunities of organizing school contests at various levels, as well as other sciences.

\subsection{Year 1987}

The first Olympiad at the national level was organized in 1987. At that time, the preuniversity educational institutions had practically no computers; first editions of the Olympiad were held based on theoretical examinations, as well as mathematical competitions. Competition topics derived from various areas of applied mathematics, theoretical computer science, logic. From edition to edition, algorithmic component became more pronounced, the subjects were moving towards practical component - to identify computer models for problem solving in various fields.

The advent of computers in schools set the beginning of a qualitatively new stage in informatics competitions. It became possible to implement the theoretical solutions in a programming language and to test these solutions. The first automated evaluation systems appeared practically immediately. 


\subsection{Year 1996}

Since 1996, students from Moldova have participated in the International Olympiad in Informatics. This also imposed adjustments to the organizational model for the Republican Olympiad in Informatics. The IOI is the reference point from which the development of all structural components of the national Olympiad flows: scientific component (competition tasks, evaluation), organizational (cultural, communication, scientific sessions), technical (computer network).

During the same period, schools were implementing individual model of using computers in labs (one student - one computer). This resulted in increase of the number of pupils fond of informatics and of programming in particular. The motivation of participants at the Olympiad increased at both national and local level. The winners of the Olympiad got national testing facilities, preferential admission to universities in the country, various awards; and a chance to compete for participation in international competitions.

\subsection{Year $2000+$}

The organizational principles and scientific assessment were finalized, supported by rules and other normative acts of the Ministry of Education. The Olympiad "matured" and continued to develop in line with the national curriculum of the computer science and the Olympic principles promoted by the Organizing Committee of the IOI. In 2004 Moldova became a member of the community of the Balkan Olympiad in Informatics and in 2007 hosted it for the first time.

In recent years the Republican Olympiad in Informatics has generated a number of local competitions organized by various educational institutions: individual and team programming competitions; online contests; ICT usage competitions; robotics competitions, etc. So, the number of students fond of various fields of Computing is growing.

\section{Moldavian Republican Olympiad in Informatics}

\subsection{The Organizational Model}

The Olympiad is held annually during the academic year: from September to May. The National Olympic Committee, the composition of which is approved annually by the Minister of Education, organizes the Olympiad. The competition is conducted in three stages: local, regional, and national (Fig. 1).

On the local and regional stage, each grade is considered a separate age group. There are two age groups on the national stage: middle school (up to $9^{\text {th }}$ grade) and high school (grades 10-12). 
Local Stage. There is a preparation period from September to December and internal competitions in educational institutions take place in January (p. 1.a and 1.b on Fig. 1).

Regional Stage. It is organized separately in each administrative unit. Teams of the educational institutions selected at the local stage participate on the regional level. Teams for the national stage are formed based on the results. To ensure more rigorous selection of the teams, the contest can take place in two rounds (p. 2.a and 2.b on Fig. 1).

National Stage. The participants are the teams selected in the previous stage and National Olympiad winners from the previous year. The total number of contestants varies between 130 and 160. Traditionally a stage lasts four days, two of which are reserved for competition. Competition sessions last between 4 and 5 hours, depending on the age group (middle school, high school) and the difficulty of the problems. After finishing the contest session, the participants can attend a self-assessment session, where they check their own solutions. Any disputes that may appear are presented to the Scientific Committee of the Olympiad. After the completion of the competition tests and resolution of disputes, the official solutions are presented. In parallel with the contest, training sessions, book launches, meetings with representatives of software companies and other activities are organized for team leaders (Fig. 1).

Winners of the national stage form the Extended National Team from which the National Team that participates in international competitions is selected (Fig. 1).

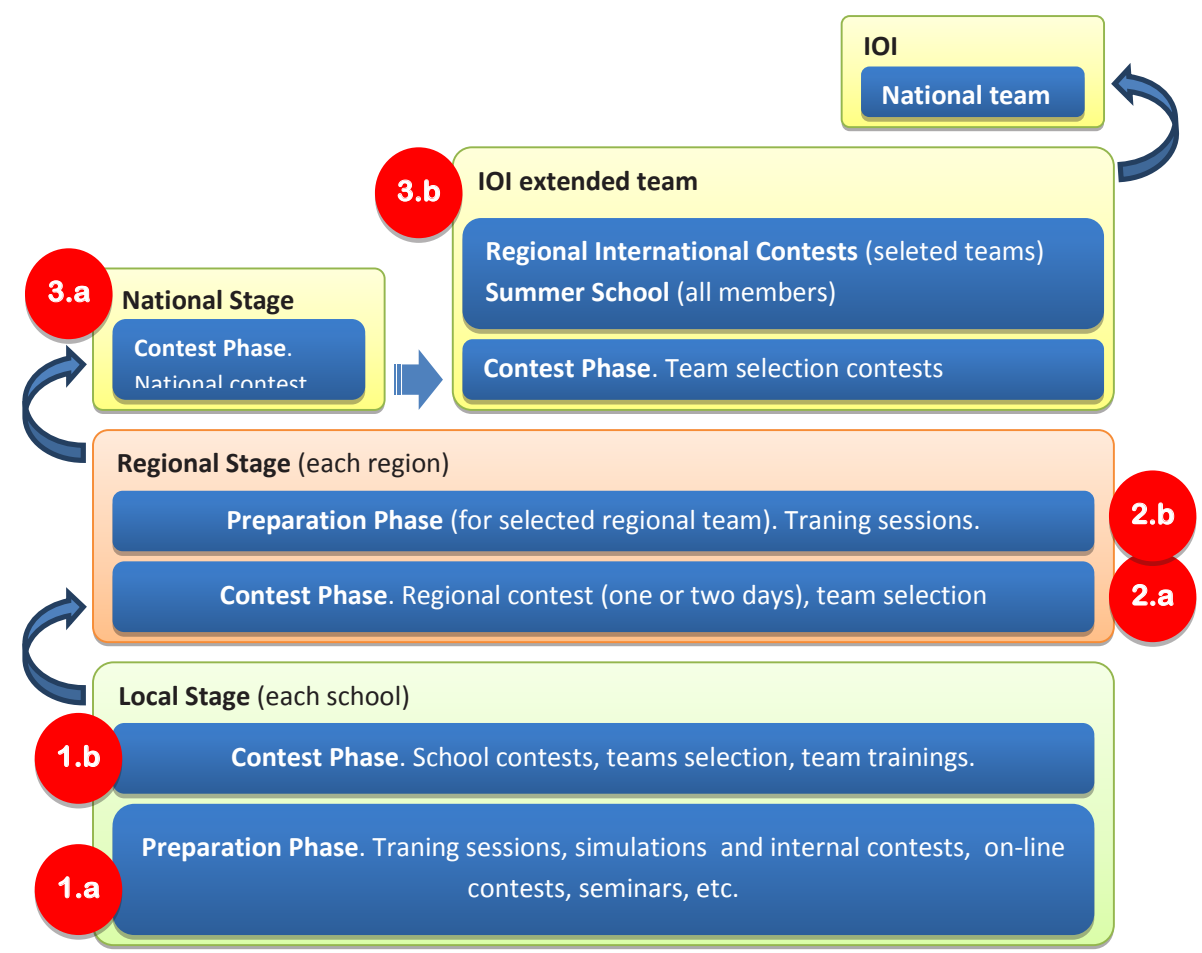

Fig.1. Stages of Republican Olympiad in Informatics. 


\subsection{Target Group}

According to the curriculum, the study of software modules begins only in the $9^{\text {th }}$ grade. However, taking into account the students' interest in programming and the capacity of self-learning of the gifted and highly gifted pupils, also younger children are accepted in the competition. The youngest participant in the history of the National Olympiad was a $6^{\text {th }}$ grade pupil (13 years old $)^{1}$.

Informatics teachers of junior grades try to identify the pupils with skills in computer science through a variety of methods and techniques. In many cases the selection is performed by the teacher of Mathematics, in the $6^{\text {th }}-7^{\text {th }}$ grades. A study conducted by the authors in $2011^{2}$ established a high degree of correlation between the performance in Mathematics and in Computer Science among the participants of the National Olympiad. Also, pupils who have good results in Physics (the study of this discipline begins in the $6^{\text {th }}$ grade) show increased interest towards programming elements. Statistics compiled over the last 12 years (Fig. 2) indicate relative stabilization in the numbers of participants in the national stage in both categories: Senior (110 on average) and Juniors (40 on average).

\subsection{Competition Topics}

Competition topics are selected according to the National Curriculum in Computer Science. The problems for Local and Regional stages are prepared separately for each grade. The problems sets for the national stage are prepared separately for the two age groups. Usually 3-4 problems are proposed in each stage, to be solved by developing programs in Pascal, C, or C++. Six problems are proposed on the national stage, in two days of competition.

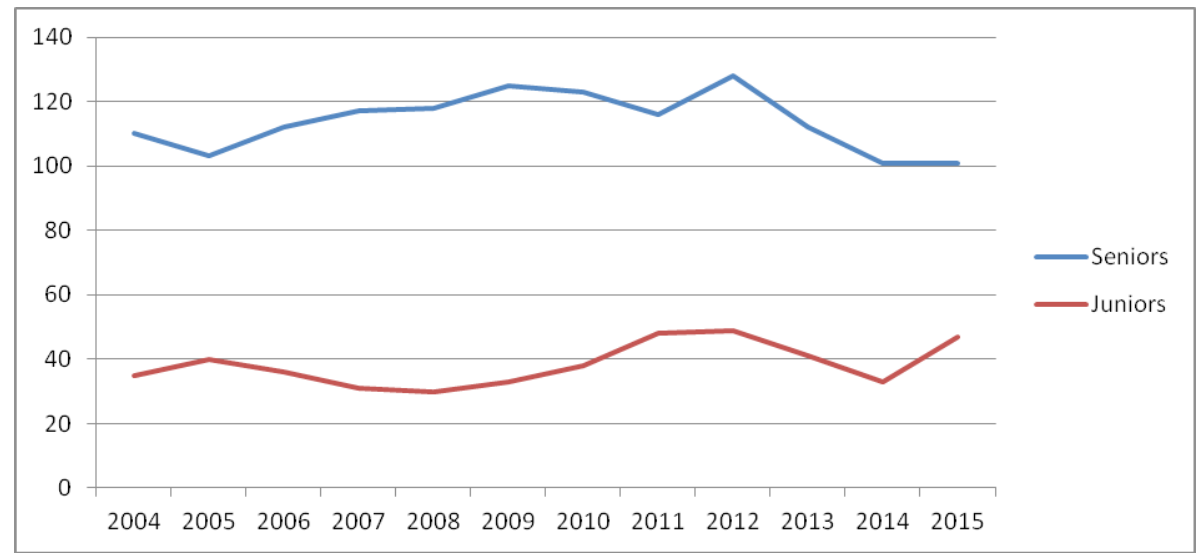

Fig. 2. Number of participants at National Stage, 2004-2015, by age categories.

1 Municipal Olympiad in Informatics, 2013

2 Didactica Pro, Nr. 4 (68), September, 2011, p.46-49 
Task topics include data structures, combinatorics, programming techniques, elements of analysis and design of algorithms, complexity, algorithms on graphs, elementary algorithms of computational geometry, mathematical modeling, heuristics, etc. The degree of difficulty and statements match the contestants' age.

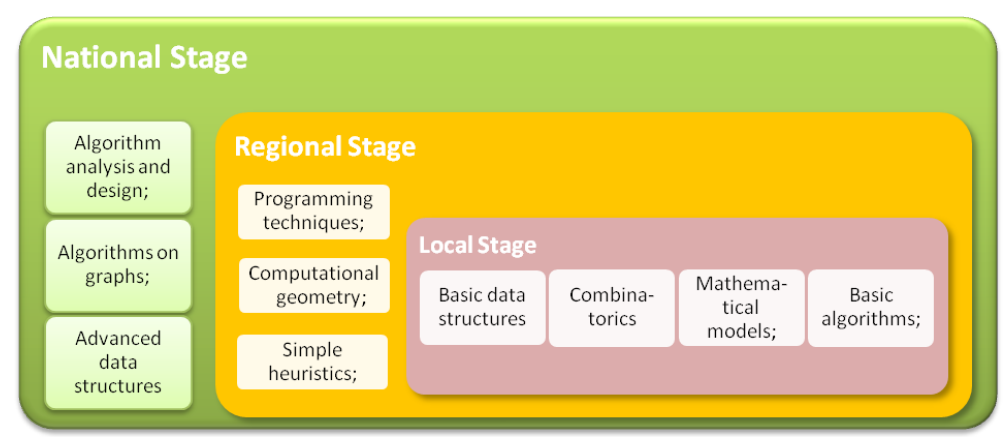

Fig. 3. The extension model of tasks domains, based on competition stages.

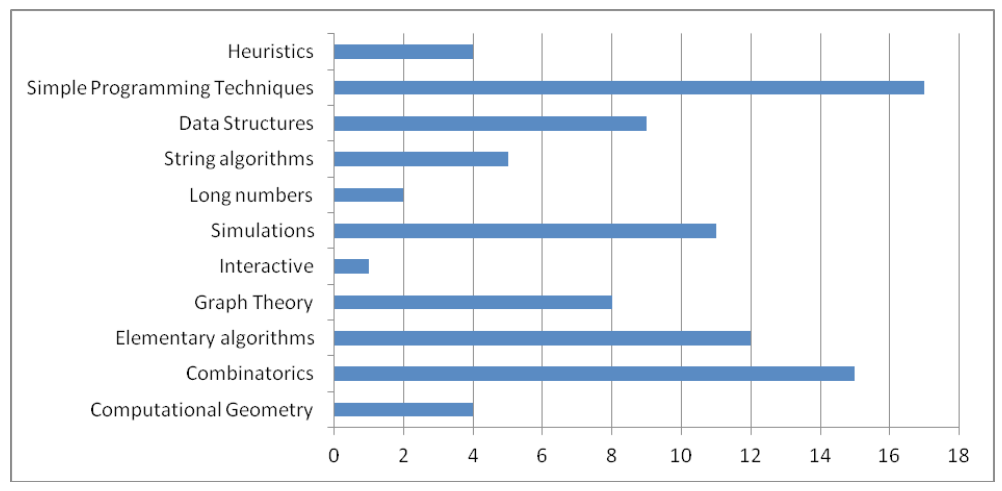

Fig. 4. Distribution of tasks by domains (last 15 years, Juniors).

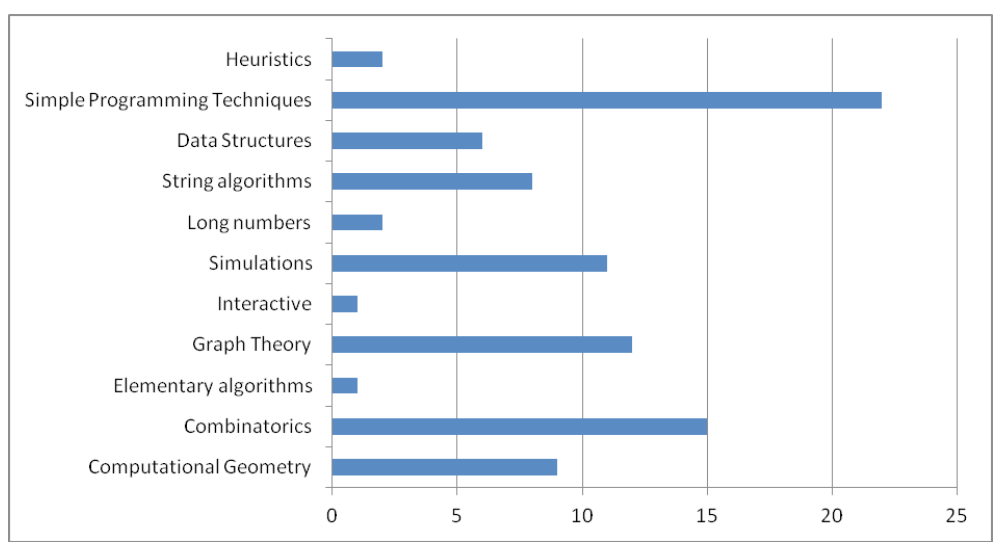

Fig. 5. Distribution of tasks by domains (last 15 years, Seniors). 
Content of tasks is developed by complexity categories, to separate competitors according to the efficiency of their solutions. The number of test cases per task ranges from 5 (local and regional stages) to 10-20 (national stage). The tests check the accuracy and efficiency of the solutions by testing special cases and enforcing time limits. The proposed model of the problem selection allows reducing the share of participants who receive zero scores. Thus, after statistical analysis of the results of the past 10 years, a quantitative distribution of the problems depending on the complexity was established (Fig. 6, 7).

Evaluation: automatic, based on a local server. Participants are identified in the system based on user accounts, generated by the system. Human intervention in the evaluation process is excluded.

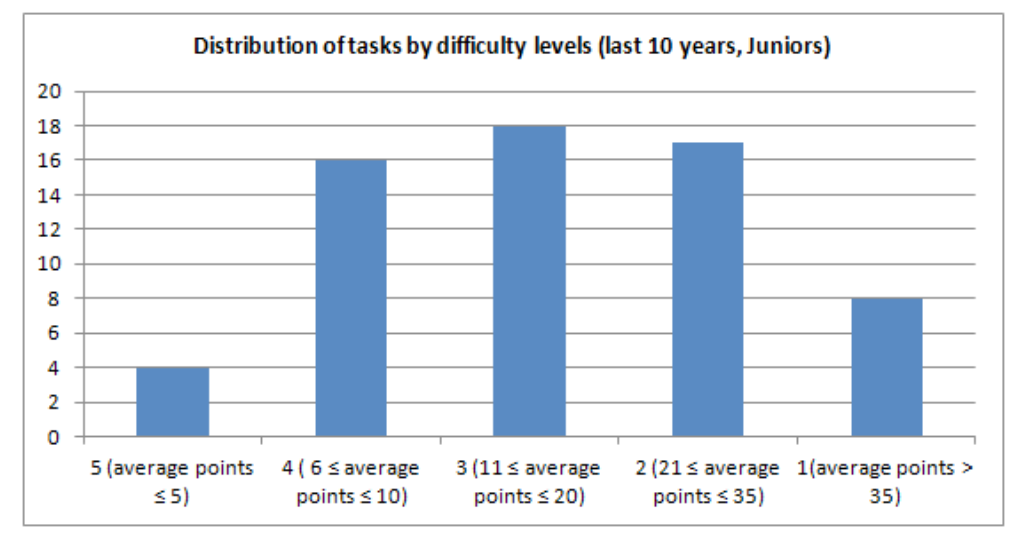

Fig. 6. Distribution of tasks by difficulty levels (last 10 years, Juniors).

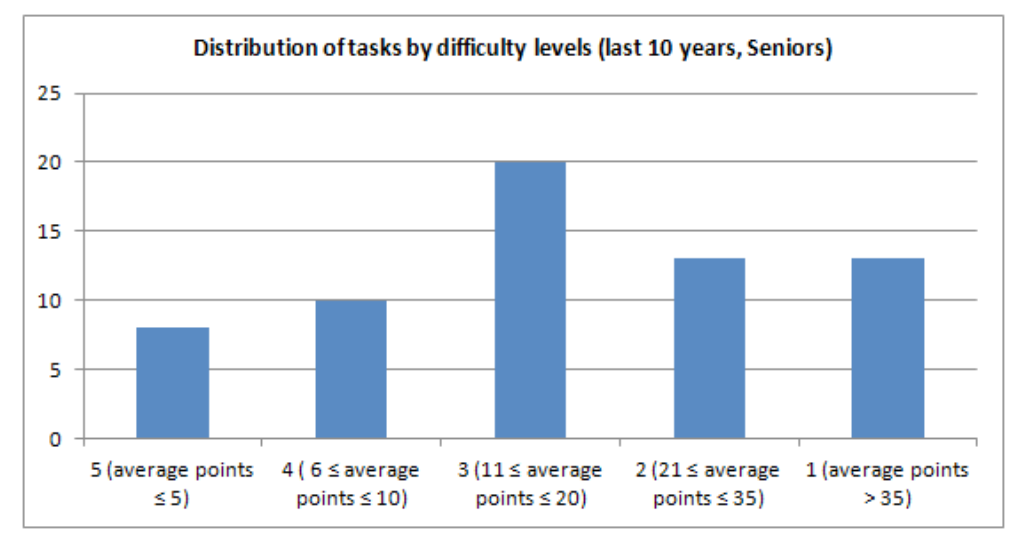

Fig. 7. Distribution of tasks by difficulty levels (last 10 years, Seniors). 


\section{Conclusions}

- The organizational model of the National Olympiad provides the opportunity to participate in the initial stage to all pupils who are interested in computer science, regardless of gender, locality, type of school, nationality.

- Distributions of the competition stages allow teams time to prepare between competitions.

- The automatic evaluation is scalable in both directions. It was used to assess the results of international competitions and is used regionally for training and regional competitions ${ }^{3}$;

- The organization of the post Olympiad activities provides qualitative preparation of National Team, as demonstrated by the results achieved by Moldova during the participation in IOI (22 bronze medals, 2 silver medals).

\section{References:}

Chistruga, Gh., Lupu, I., Gremalschi, A. (2015). Didactic digital supports for preparation in informatics. In: Proceedings of The National Scientific Conference "85 Years of Higher Education in Moldova", Chisinau, 24-25 September, 2015. 117-122.

Gremalschi, A. (2001, 2002, 2003, 2004, .., 2013). Republican Olympiad in Informatics. Tasks and solutions. 2001 (2002, 2003, 2004, ..., 2013). Chisinau, ASEM.

Prisacaru, A., Besliu, V., Bolun, I., et. al. (2014). Republican Olympiad in Informatics. Tasks and solutions. Chisinau.

Prisacaru, A., Besliu, V., Bolun, I. et al. (2015). Republican Olympiad in Informatics. Tasks and solutions. Chisinau.

Gremalschi, A., Corlat, S. (2011). The role of interdisciplinary in mathematics-informatics in preparing students performance. Didactica Pro, 4(68), 46-49.

Informatics. Curriculum for Secondary Education, High School. (2010). Chisinau, Stiinta.

Informatics. Curriculum for Secondary Education, Gymnasium. (2010). Chisinau, Lyceum.

\footnotetext{
3 Proceedings of the national scientific conference " 85 years of higher education in Moldova", Chisinau, 24-25 September, 2015.
} 


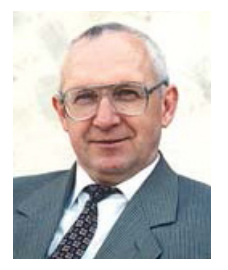

A. Gremalschi, PhD, professor, Technical University of Moldova; Director, Institute for Public Policy.

Author of textbooks in Computer Science.

Coordinator of educational projects.

Organizer of National Olympiads in Informatics (since 1997).

Leader of national team on Balkan Olympiad in Informatics, International Olympiad in Informatics (since 1996).

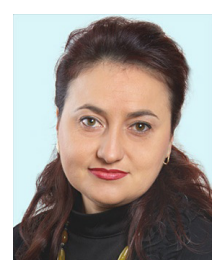

A. Prisacaru, consultant, Department of Pre-University Education, Ministry of Education of the Republic of Moldova.

Professor of Informatics, MSc in Informatics.

Organizer of the summer camps for preparing the Olympic teams.

Organizer of National Olympiads in Informatics (since 2013).

Leader of national team on Balkan Olympiad in Informatics (2014), International Olympiad in Informatics (2015).

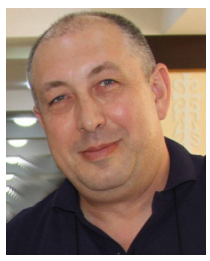

S. Corlat, MSc in Exact Sciences. Lecturer at Tiraspol State University. Research areas: computational geometry, graph theory, computer graphics. Also involved in institutional, national and international projects for e-Learning. Trainer of Informatics team of "Orizont" Lyceum. Leader of national team on Balkan Olympiad in Informatics $(2003,2004,2006,2009)$, International Olympiad in Informatics (2014, 2015). 